\title{
Neuropathic bladders: Investigation and treatment through their lifetime
}

\author{
Peter D. Metcalfe, MD, MSc \\ University of Alberta, Stollery Children's Hospital, Edmonton, AB, Canada
}

Cite as: Can Urol Assoc J 2017;11 (1-2Suppl1):S81-6. hittp://dx.doi.org/10.5489/cuaj.4276

See related commentary on page $\$ 87$.

\section{Abstract}

The child with a neuropathic bladder requires lifelong dedicated care. Just as each patient presents with unique physiology, each phase of their life presents varying challenges. The primary concern for our patients is their renal health, but continence and independence also play significant roles. Most patients can be managed conservatively, but a myriad of surgical options are also available, reinforcing our emphasis on individualized care. Appropriate presurgical planning is required to ensure the right patient receives the best operation for his/her wants and needs. Furthermore, the numerous potential complications must be understood and longterm followup and surveillance is required.

This review outlines the basic pathophysiology, investigations, and treatments, with a focus on the changing needs throughout their lives.

\section{Introduction}

Treatment of neuropathic bladders remains one of the challenges of pediatric urology. However, despite the time invested and advances made, outcomes are often not ideal and care can be frustrating. Historically, children with neurogenic bladders would go on to renal failure and suffer a significantly reduced life expectancy. ${ }^{1,2}$ However, proper management, treatment, and expectations can result in excellent health and improvements in quality of life. Although the classic example is the child with a myelomeningocele, a significant number of our patients have other spinal cord defects, including lipomyelomeningocele, fatty filum, and occult tethered cords.

Children with neuropathic bladders require lifelong care and individualized goals. Due to the diversity of pathophysiology, comorbidities, and expectations, each patient must be thoroughly and independently evaluated. One of the unique aspects of pediatric urological care is the tailoring of care to the development of the child. Family and patient expectations can change significantly through the newborn, preschool, elementary, adolescent, and adult periods. Optimal management requires comprehensive knowledge of the family, pathophysiology, and the full complement of treatment options.

The intent of this review is to outline the investigation and treatment options for patients with a neuropathic bladder, with an emphasis on treatment goals as they progress through their lifetime.

\section{Prenatal}

A significant number of our patients are diagnosed prenatally with a spinal cord defect. Modern second trimester ultrasonography techniques are very accurate in the determination of spinal defects and in differentiating an open vs. closed spinal cord lesion. ${ }^{3-5}$ If results are not diagnostic, magnetic resonance imaging (MRI) is often used. ${ }^{6}$

Although obviously an emotional diagnosis, it affords the family an opportunity to prepare themselves for the challenges ahead. Although controversial, in-utero closure of the open neural tube defect may offer some advantages to the child, but the risks of preterm labor $(13 \%$ delivered at less than 30 weeks gestation) and maternal health remain significant concerns. ${ }^{7-9}$ An ambitious randomized trial of 183 patients has demonstrated decreased need for ventriculoperitoneal (VP) shunts, ${ }^{7}$ as well as improved mental and motor development, decreased hind brain herniation, and improved ambulation by 30 months. ${ }^{9}$ Urological outcomes were not part of the initial assessment, but a 2015 review of the Management of Myelomeningocele Study (MOMS) demonstrated no difference in the need for clean intermittent catheterization $(\mathrm{CIC})$, but patients appeared to have less bladder trabeculation and fewer open bladder necks. ${ }^{10}$ The long-term implications of this are unknown, but after a review of their results, the Children's Hospital of Philadelphia declared that $18.5 \%$ of children were toilet-trained, although they did not elaborate on controls. ${ }^{11}$ This cohort suffered 
a $7 \%$ neonatal mortality rate. ${ }^{11}$ Another prospective study failed to show any benefit on the urinary tract, with over $90 \%$ of patients demonstrating urinary tract dysfunction on urodynamics in the first year. ${ }^{12}$ Prenatal intervention should currently be considered only in the context of clinical trials in specialized centres, where the expertise exists to minimize the risks to mother and fetus.

\section{Neonatal and infant}

After prenatal diagnosis, the newborn should be delivered in a tertiary-care pediatric hospital with ready access to a highlevel neonatal intensive care unit, pediatric neurosurgery, and pediatric urology expertise. The initial surgery, which includes closure of the spinal cord defect and determination of the need for a VP shunt, is performed by a neurosurgeon.

Commonly, after newborn closure, the babies have an indwelling urethral catheter and are maintained in a lateral decubitus position to protect the surgical wound. Therefore, immediate assessment of the urinary tract is difficult. Once the baby is able to be maintained in a supine position, the initial investigation of the urinary tract includes an ultrasound to determine the presence of hydronephrosis and either $\mathrm{CIC}$ or bladder scan to determine the presence of urinary retention.

Proactive management of the newborn was nicely summarized by Snow-Lisy et al as either expectant or proactive. ${ }^{13}$ Expectant management involves regular monitoring with clinical assessment and ultrasound and the introduction of $\mathrm{CIC}$, urodynamics, or anticholinergics if deterioration occurs. Although proponents argue that the early detection of upper tract changes results in reversibility, it may result in a higher rate of bladder augmentation. ${ }^{14}$

Expectant management involves early institution of $\mathrm{CIC}$ and the early and regular use of urodynamic testing to guide therapy. The arguments for early use of CIC is supported by moderately strong basic and clinical evidence concluding improved long-term bladder and renal health. ${ }^{15}$ Proponents also argue that $\mathrm{CIC}$ is better tolerated by the patient and family when introduced early. Regular (every six months or annually) urodynamics will also identify the neurogenic overactive bladder, whereby the resultant early introduction of anticholinergic therapy may further improve long-term outcomes. ${ }^{15}$

At our institution, we feel that early adoption of $\mathrm{CIC}$ is beneficial to the patient for long-term outcomes and for the parents with respect to compliance and tolerance. We would rather withdraw $\mathrm{CIC}$ if the bladder is emptying well than try to institute it later, after the development of infections or hydronephrosis. We record pre-catheterization residuals in the neonatal intensive care unit (NICU) and once the patient is at home, in order to adjust the frequency of catheterization accordingly. If catheterized volumes are consistently
$<30 \mathrm{ml}$, we decrease the frequency of catheterization from four to three times daily, with further decreases if residuals remain $<30 \mathrm{ml}$.

The family's first visit to our spina bifida clinic is at three months of age, then at six months and at six-month intervals for the first few years of life. Patients undergo a comprehensive assessment by: urology, neurosurgery, physiatry, physiotherapy, and gait and wheelchair seating in older children. Psychology and social work are also available and play a prominent role.

We obtain our first urodynamics at six months of age and annually thereafter. If these demonstrate poor compliance and elevated detrusor leak point pressures or end-filling pressures, we institute oxybutynin at $0.2 \mathrm{mg} / \mathrm{kg}$ three times daily and ensure compliance with catheterization four times daily. However, if baseline pressures remain low, but the child has significant neurogenic overactivity, we also consider instituting oxybutynin. The main indication with the latter scenario would be to prevent long-term deterioration, as opposed to the former, whereby we would consider the kidneys to be at significant risk from the elevated pressures and that the pressures need definite, immediate attention. If medications have been initiated, we advocate for followup urodynamics in 3-6 months to assess treatment effects.

If the initial ultrasound demonstrates bilateral hydroureteronephrosis, especially with elevated pressures on urodynamics, we consider more aggressive intervention. It becomes necessary to know the volumes attained at catheterization. This can then be correlated to the cystometrogram and the pressures that are recorded when these volumes occur. Therefore, the urology team can assess whether or not it is practical to catheterize at volumes low enough to maintain safe pressures. Anticholinergic medications can be titrated up until side effects are seen. If the volumes at which the elevated pressures are occurring are seen at volumes where catheterization frequency is unrealistic, or the parents are unable to perform $\mathrm{CIC}$, a vesicostomy is a very effective option. ${ }^{16}$

\section{Preschool}

These years tend to be relatively simple from a urology perspective. The primary focus is renal health and the high-risk patients have likely declared themselves and have undergone treatment. The risk of deterioration is low. Some families will want to pursue continence, which is always encouraged with appropriate expectations.

Treatment of urinary tract infections (UTIs) in this age group will not vary much compared to the general population. It is fundamentally important that all caregivers for the child are able to differentiate between asymptomatic colonization, which will be universal, and a symptomatic UTI.

A recent study found the incidence of UTI was approximately $35 \%$ in infants undergoing $\mathrm{CIC}$ and $18 \%$ in those 
who were voiding spontaneously. ${ }^{17}$ We advocate against screening urine cultures, as treatment of asymptomatic bacteriuria is not indicated. The use of prophylactic antibiotics is controversial, and although used frequently with anecdotal benefits, a randomized trial from Hong Kong did not show any benefit. ${ }^{18}$ Many patients may continue to suffer from UTIs despite oral prophylaxis and these patients may benefit from an intravesical aminoglycoside. The use of intravesical gentamycin is well-documented, with excellent safety and efficacy, ${ }^{19,20}$ including in patients with enterocystoplasty. ${ }^{21}$ No series have demonstrated detectable serum levels, resulting in an exceptional safety profile. Although multiple dosing regimens have been used, many (including ours) prescribe $480 \mathrm{mg}$ of gentamycin in $1 \mathrm{~L}$ of normal saline, with $30 \mathrm{cc}$ administered per day ( $14 \mathrm{mg} /$ day). Many pharmacies will dispense in a $30 \mathrm{cc}$ prefilled syringe, greatly facilitating administration.

The incidence of infections may also be related to vesicoureteral reflux (VUR). In addition to the normal ureterovesical junction (UVJ) insertion, VUR is also related to bladder pressures; it should be assessed at the regular video-urodynamics, whereby the detrusor pressure, when reflux occurs, can be helpful in planning treatment. This is critical because if VUR is managed surgically with a high-pressure bladder, the treatment is more likely to fail. Its presence increases the risk of UTI to approximately $50 \% .{ }^{17}$ Treatment is primarily focused on minimizing bladder pressures, but if reflux and infection continue despite good medical control, or videourodynamics demonstrate VUR at low pressures, surgical correction may be required. Indications for surgery will not vary from non-neuropathic patients.

Although most children will potty-train at ages $2-3$, this is much less likely in our patients with a spinal dysraphism. We never discourage families, but will temper expectations. If the caregivers are interested, we will start by having the child sit on the toilet every two hours on a scheduled basis.

A review of our pediatric clinic data demonstrated that only $5 \%$ of our lumbar myelomeningocele patients were able to void spontaneously and were considered continent; this increased to $30 \%$ in the sacral myelomeningocele population. In our closed neural tube defect population (lipomyelomeningocele and tethered cord), rates were just above $50 \%$. Fecal continence was achieved with conservative measures in approximately two-thirds of our patients. ${ }^{22}$

\section{Elementary school}

As the child approaches the age of five years, continence becomes an increasing concern. Some patients will be continent between catheterization so no further intervention is necessary. If the child is still wet, we will start with oxybutynin $0.2 \mathrm{mg} / \mathrm{kg}$ three times daily, up to $5 \mathrm{mg}$ three times daily. Side effects are common, but are usually tolerable.
These include dry mouth, decreased sweating, facial flushing, worsening constipation, and somnolence. Oxybutynin is readily available in a suspension, facilitating administration. Often doses are increased well beyond the $0.2 \mathrm{mg} / \mathrm{kg}$ dose and titrated to effect or intolerance. It is also often required to increase the frequency of daytime catheterization to ensure the bladder is empty before reaching elevated pressures or undergoing volume-related contractions.

As always, persistent incontinence requires a careful history to identify other potential barriers or triggers. For example, caffeinated drinks, constipation, or inadequate access to appropriate aids or bathrooms can all promote incontinence.

Newer formulations of anticholinergic medications, while not approved for use in children, are regularly used in our clinic to improve compliance and quality of life for families. Oxybutynin gel can work very well as a oncedaily application and solifenacin can be crushed and taken orally. Once the patient is able to tolerate swallowing pills, the long-acting formulations can be very helpful in reducing side effects and increasing alternatives. Dr. Bolduc has pioneered increasing dosing and combining antimuscarinics to increase efficacy and minimize side effects. ${ }^{23}$ Once adult dosing has been reached with a single medication, a second antimuscarinic is well-tolerated and may result in continence or safer bladder pressures. ${ }^{23}$

In our patients who have persistent hydronephrosis despite maximal medical management, overnight catheter drainage can be attempted. Since the highest bladder volumes, and therefore pressures, will likely occur overnight, continuous overnight drainage may decrease renal stress. Koff et al reported that overnight catheterization decreased hydronephrosis and increased bladder capacity. ${ }^{19} \mathrm{Although}$ many patients do not tolerate this, due to the catheter disconnecting during sleep, urethral trauma, and a significant "nuisance factor," many families appreciate it as an effective means to prevent surgical intervention.

If maximal medical management remains inadequate, surgical options may be entertained. Urodynamics and a focused history are critical in assessing the causes of the incontinence, whether it is secondary to an inadequate sphincter complex, neurogenic detrusor overactivity, or poor compliance.

Minimally invasive options are available and include intravesical onabotulinumtoxinA (Botox ${ }^{\circledR}$ ) injections and bladder neck injections. OnabotulinumtoxinA has good efficacy, but will require repeated injections, and although some of our patients can tolerate this under local anesthesia, regular general anesthetics may dissuade many families. Mildly incompetent bladder necks may respond to injection of bulking agents such as domperidone (Deflux ${ }^{\circledR}$ ), but expectations should be low. ${ }^{24}$

Our first priority is always renal health; therefore, surgical intervention becomes mandatory if hydronephrosis persists 
or renal insufficiency appears. The major consideration is whether or not the patient and family are willing and able to undergo continent reconstruction or whether they will do better with an incontinent diversion.

Many of our patients and families are not able to catheterize sufficiently to minimize bladder pressures and renal stress. Therefore, an incontinent diversion is required; alternatives include a vesicostomy or an ileovesicostomy/ileal chimney. As the child grows, the vesicostomy is often unable to provide reliable drainage, as the increasing distance between pelvis and stoma result in tension and stenosis. Controlling urinary drainage with an incontinence product also becomes more difficult. Older children often require an ileovesicostomy, which has the added advantage of a urostomy and bag, duecreasing odors and eliminating the need for incontinence products. ${ }^{3,5}$

However, if the patient and family are known to be reliable and motivated to achieve continence, they may prove to be candidates for a continent reconstruction. This is a major surgery, but more importantly, requires an enormous commitment from the patient and family for their entire lives. Although definite improvements in quality of life have been difficult to demonstrate with cohort studies, ${ }^{4,25}$ many families are very keen on proceeding.

These reconstructive procedures require significant preoperative planning and counselling. The surgeon should consider four separate aspects when developing a plan: 1) adequacy of bladder neck/sphincter complex; 2) need for increased bladder capacity; 3) desire for a cutaneous catheterizable channel; and 4) fecal continence procedures. It is of fundamental importance not to pursue any reconstruction without significant planning, both from a surgical and caregiver perspective. If even the best operation is performed in the wrong patient, the results can be disastrous. ${ }^{6,26,27} \mathrm{It}$ has been shown that the most important factor in successful outcomes is proper family selection. . $^{7-9,28}$

Bladder neck procedures range from endoscopic injections of the bladder neck to outpatient suburethral slings, to bladder neck reconstruction, to ligation or closure of the bladder neck. ${ }^{7,29}$ Many pediatric urologists prefer a moderate approach, to leave the patient with a "pop-off" mechanism in the case of inability or unwillingness to catheterize. If an aggressive approach is considered (bladder neck reconstruction, occlusive sling, bladder neck closure) the patient will require a continent cutaneous catheterizable channel.

The most common means of increasing bladder capacity and improving compliance is augmentation with ileum; however, a myriad of other tissues have been used with varying degrees of succes. ${ }^{9,26} \mathrm{~A}$ review of 500 patients from Indiana University demonstrated a $34 \%$ need for subsequent surgery (Fig. 1). ${ }^{10,26}$ Following bladder augmentation, patients must catheterize regularly and perform bladder irrigations with water to minimize infection risk and prevent bladder stone formation. Routine yearly ultrasounds are important to detect stones in asymptomatic patients and allow for prompt intervention.

Complications range in severity from increased frequency of UTIs, to bladder stones, to death. If an augmented bladder is chronically distended or subject to significant forces, perforation can occur. This can result in life-threatening sepsis due to peritonitis. In a large series from Indiana, perforations occurred in $8.6 \%$ of patients, with one death. ${ }^{11,30}$ Due to the critically ill presentation of most patients, immediate laparotomy with peritoneal washout, primary closure, and maximal drainage is required. If the patient is not ill and has a small, contained leak, conservative management with percutaneous drains and aggressive bladder drainage can be successful. ${ }^{11,27}$ Most perforations occur in the posterior aspect of the bladder-bowel anastomosis and, therefore, identification and repair may require bivalving the bladder completely. ${ }^{12,31}$ Worse still is that due to the neurogenic situation, the initial injury may go unnoticed and result in a late presentation. Therefore, a high index of suspicion is required once a patient has had an augmentation.

There has also been concern regarding death from malignancy after bladder augmentation, which is reported to be $0-5 \%,{ }^{13,32}$ and is likely highest when gastric segments are used. ${ }^{14,33}$ However, it is unclear whether an augmentation increases this risk or if it is the nature of the neuropathic bladder 
(i.e., exstrophy, immune suppression after transplant, smoking, infection, catheterization, chronic UTIs, and bladder stones). ${ }^{15,34}$ Unfortunately, screening with cystoscopy and urinalysis has not proven to reduce mortality. ${ }^{16,32,34}$

Although many patients prefer to continue catheterizing per urethra, a catheterizable abdominal wall stoma can be an attractive option. If an aggressive approach to the bladder neck is required, the patients may no longer be able to catheterize per urethra. Furthermore, the wheelchair-dependent patient, particularly females, may no longer need to lie on the bathroom floor and catheterize and many patients prefer the modesty gained by not having an aide expose their genitalia to catheterize.

Complications from channels are also common, ranging from $5-25 \%$, and primarily include difficulty catheterizing or incontinence per channel. ${ }^{17,35,36}$ Minor, outpatient skin stoma revisions or injections of bulking agents into the channel are moderately successful. ${ }^{18,36}$

The importance of discussing and achieving fecal continence cannot be overemphasized, especially if the child is undergoing surgery for urinary continence. The success rates of antegrade fecal enemas, either a Malone antegrade continence enema (MACE) or cecostomy tube, mandate their inclusion in preoperative discussions. ${ }^{19,20,37,38}$ The choice between one or the other is primarily patient-driven, the primary factor being the external "button" compared to an abdominal catheterizable stoma. The cecostomy tube is usually inserted percutaneously by radiology, with two stages, compared to an open/laparoscopic MACE procedure. A series at the University of Alberta showed similar revision rates and satisfaction with both. ${ }^{21,39}$

Obviously, the entire spectrum of lower urinary tract reconstruction is beyond the scope of this review, but we believe that all urologists should be aware of the generalities and potential life-threatening complications. This is becoming ever more important, as our patients are living through adulthood, and the transition of their care from pediatric hospitals and caregivers is an enormous issue.

\section{Adolescence}

Adolescence can be a difficult time for these patients. They are subject to the same quests for independence and rebelliousness as any teenager and as seen across any chronic health condition. Their medical challenges can take an emotional toll and the social consequences of their mobility, cognitive, and continence can be devastating. Incontinence can result in patients becoming ostracized or bullied. However, their cognitive challenges can limit their ability to independently maintain continence and may result in resentment, frustration, or apathy. This ultimately results in inadequate care. As described above, this can be devastating in patients who have had complicated reconstruction, whereby if strict catheterizing regimens are not adhered to, complications are common and significant. Therefore, the urologist and all caregivers must be on alert for the adverse consequences along this journey to independence.

Another unique aspect to the adolescent patient with a neuropathic bladder is the advent of sexuality. This is a poorly studied area, but work from Toronto highlighted the lack of education and desire to learn more from their healthcare providers. ${ }^{17,40}$ There are a few reports of adults with significant impairments, with one from Boston stating a male erectile dysfunction rate of $59 \%$ and female dysfunction at $89 \%$. However, the response rate was $30 \%$ and dysfunction varied with the level of the lesion. ${ }^{23,41}$

\section{Adult}

The improved care of these patients has resulted in a drastic increase in the life expectancy. Unfortunately, however, this has also resulted in a significant gap in healthcare. ${ }^{42}$ Whereas most tertiary centres have a well-established pediatric multidisciplinary clinic, the care of the adult spina bifida patient falters. Many children's hospitals focus on transitioning the care to an appropriate adult provider and guidelines are beginning to emerge to facilitate this..$^{23,43}$ Several Canadian centres, including in Edmonton and Halifax, have independent adult spina bifida clinics, which closely resemble their pediatric model.

Although many of their neurosurgical and orthopedic issues have quiesced, as they are no longer growing and most are wheelchair-dependent, the urological problems of these adult patients remain at the forefront. Incontinence and high bladder pressures are very prevalent, especially since many are without their parental supports. Although surgical intervention is very prevalent at this age, endoscopic revisions to continent diversions and bladder stones account for a majority of the cases. My personal experience is that catheterizing becomes an increasing burden and many patients look towards converting to an incontinent diversion.

Dr. Doug Hussman has published several concerning reports regarding long-term compliance and risks of renal insufficiency in patients who have had prior reconstructions. ${ }^{44} \mathrm{He}$ also believes that an incontinent diversion is preferred in the adult with a neuropathic bladder, as many are unable to provide sufficient self-care to ensure their longterm health. ${ }^{44} \mathrm{He}$ is an ardent supporter of management with a suprapubic tube as the simplest means of maximizing renal preservation.

\section{Conclusion}

The patient with spina bifida requires a lifetime of complex and intensive urological care. We have great potential do improve their quality of life, but this requires a comprehensive 
and accurate assessment of the patient's wishes and abilities. We must never ignore the significant complications that can occur and must be vigilant to ensure supportive followup.

Competing interests: The author reports no competing personal or financial interests.

This paper has been peer-reviewed.

\section{References}

1. Singhal B, Mathew KM. Factors affecting mortality and morbidity in adult spina bifida. Eur J Pediatr Surg 1999;9Suppl1:31-2. https://doi.org/10.1055/s-2008-1072310

2. Oakeshott P, Reid F, Poulton A, et al. Neurological level at birth predicts survival to the mid-40s and urological deaths in open spina bifida: A complete prospective cohort study. Dev Med Child Neurol 2015. [Epub ahead of print]. https://doi.org/10.1111/dmcn.12698

3. Hellenthal NJ, Short SS, O'Connor RC, et al. Incontinent ileovesicostomy: Long-term outcomes and complications. Neurourol Urodyn 2009;28:483-6. https://doi.org/10.1002/nau.20695

4. Coleman BG, Langer JE, Horii SC. The diagnostic features of spina bifida: The role of ultrasound. Fetal Diagn Ther 2015;37:179-96. https://doi.org/10.1159/000364806

5. Ching $C B$, Stephany HA, Juliano $T M$, et al. Outcomes of incontinent ileovesicostomy in the pediatric patient. J Urol 2014;191:445-50. hitps://doi.org/10.1016/i.juro.2013.08.008

6. Aaronson OS, Hernanz-Schulman M, Bruner JP, et al. Myelomeningocele: Prenatal evaluation — comparison between transabdominal US and MR imaging. Radiology 2003;227:839-43. https://doi.org/10.1148/radiol.2273020535

7. Tulipan N, Wellons JC, Thom EA, et al. Prenatal surgery for myelomeningocele and the need for cerebrospinal fluid shunt placement. J Neurosurg Pediatr 2015;16:613-20. https://doi.org/10.3171/2015.7.PEDS15336

8. Grivell RM, Andersen C, Dodd JM. Prenatal vs. postnatal repair procedures for spina bifida for improving infant and maternal outcomes. Cochrane Database Syst Rev 2014;10:CD008825. https://doi.org/10.1002/14651858.cd008825.pub2

9. Adzick NS, Thom EA, Spong CY, et al. A randomized trial of prenatal vs. postnatal repair of myelomeningocele. N Engl J Med 2011;364:993-1004. hitps://doi.org/10.1056/NEJMoa1014379

10. Brock JW, Carr MC, Adzick NS, et al. Bladder function after fetal surgery for myelomeningocele. Pediatrics 2015;136:e906-13. https://doi.org/10.1542/peds.2015-2114

11. Carr MC. Urological results after fetal myelomeningocele repair in pre-MOMS trial patients at the Children's Hospital of Philadelphia. Fetal Diagn Ther 2015;37:211-8. https://doi.org/10.1159/000362932

12. Leal da Cruz $M$, Liguori R, Garrone $G$, et al. Categorization of bladder dynamics and treatment after fetal myelomeningocele repair: First 50 cases prospectively assessed. J Urol 2015;193:1808-12. https://doi.org/10.1016/i.juro.2014.10.118

13. Snow-Lisy DC, Yerkes EB, Cheng EY. Update on urological management of spina bifida from prenatal diagnosis to adulthood. J Urol 2015;194:288-96. https://doi.org/10.1016/i.juro.2015.03.107

14. Kaufman AM, Ritchey ML, Roberts AC, et al. Decreased bladder compliance in patients with myelomeningocele treated with radiological observation. J Urol 1996;156:2031-3. https://doi.org/10.1016/ S0022-5347(01)65427-2

15. Kaefer M, Pabby A, Kelly $M$, et al. Improved bladder function after prophylactic treatment of the high-risk neurogenic bladder in newborns with myelomeningocele. J Urol 1999;162:1068-71 https://doi.org/10.1016/S0022-5347(01)68069-8

16. Mandell J, Bauer SB, Colodny AH, et al. Cutaneous vesicostomy in infancy. J Urol 1981;126:92-3.

17. Kaye IY, Payan M, Vemulakonda VM. Association between clean intermittent catheterization and urinary tract infection in infants and toddlers with spina bifida. J Pediatr Urol 2016. [Epub ahead of print]. https://doi.org/10.1016/i.jpurol.2016.02.010

18. Clarke SA, Samuel M, Boddy S-A. Are prophylactic antibiotics necessary with clean intermittent catheterization? A randomized, controlled trial. J Pediatr Surg 2005;40:568-71. https://doi.org/10.1016/i ipedsurg.2004.11.027

19. van Nieuwkoop C, Exter den PL, Elzevier HW, et al. Intravesical gentamicin for recurrent urinary tract infection in patients with intermittent bladder catheterization. Int J Antimicrob Agents 2010;36:485-90. https://doi.org/10.1016/i.jantimicag.2010.05.005
20. Wan J, Kozminski M, Wang SC, et al. Intravesical instillation of gentamicin sulfate: In vitro, rat, canine, and human studies. Urology 1994;43:531-6. https://doi.org/10.1016/0090-4295(94)90249-6

21. DeFoor W, Ferguson D, Mashni $S$, et al. Safety of gentamicin bladder irrigations in complex urological cases. J Urol 2006;175:1861-4. https://doi.org/10.1016/S0022-5347(05)00928-6

22. Metcalfe $P$, Gray $D$, Kiddoo D. Management of the urinary tract in spina bifida cases varies with lesion level and shunt presence. J Urol 2011;185:2547-51. https://doi.org/10.1016/i.jur0.2011.01.025

23. Nadeau G, Schröder A, Moore K, et al. Double anticholinergic therapy for refractory neurogenic and nonneurogenic detrusor overactivity in children: Long-term results of a prospective open-label study. Can Urol Assoc J 2014;8:175-80. https://doi.org/10.5489/cuaj.1362

24. Halachmi $S$, Farhat $W$, Metcalfe $P$, et al. Efficacy of polydimethylsiloxane injection to the bladder neck and leaking diverting stoma for urinary continence. J Urol 2004;171:1287-90. https://doi.org/10.1097/01.ju.0000111221.43756.03

25. MacNeily AE, Jafari S, Scott $\mathrm{H}$, et al. Health-related quality of life in patients with spina bifida: A prospective assessment before and after lower urinary tract reconstruction. J Urol 2009; 182:1984-91. https://doi.org/10.1016/i.juro.2009.02.060

26. Metcalfe PD, Rink RC. Bladder augmentation: Complications in the pediatric population. Curr Urol Rep 2007;8:152-6. https://doi.org/10.1007/s1 1934-007-0065-x

27. Metcalfe PD, Casale AJ, Kaefer MA, et al. Spontaneous bladder perforations: A report of 500 augmentations in children and analysis of risk. J Urol 2006;175:1466-70. https://doi.org/10.1016/ S0022-5347(05)00672-5

28. Clark T, Pope JC, Adams MC, et al. Factors that influence outcomes of the Mitrofanoff and Malone antegrade continence enema reconstructive procedures in children. J Urol 2002;168:1537-40. https://doi.org/10.1016/S0022-5347(05)64515-6

29. Cole EE, Adams MC, Brock JW, et al. Outcome of continence procedures in the pediatric patient: A single institutional experience. J Urol 2003;170:560-3. https://doi.org/10.1097/01.ju.0000078015.55801.52

30. Metcalfe $\mathrm{PD}$, Cain $M P$, Kaefer $M$, et al. What is the need for additional bladder surgery after bladder augmentation in childhood? J Urol 2006;176:1801-5. https://doi.org/10.1016/i.juro.2006.03.126

31. Anderson PA, Rickwood AM. Detrusor hyper-reflexia as a factor in spontaneous perforation of augmentation cystoplasty for neuropathic bladder. Br J Urol 1991;67:210-2. https://doi.org/10.1111/j.1464410X.1991.tb15112.x

32. Biardeau $X$, Chartier-Kastler $E$, Rouprêt $M$, et al. Risk of malignancy after augmentation cystoplasty: A systematic review. Neurourol Urodyn 2016;35:675-82. https://doi.org/10.1002/nau.22775

33. Husmann DA. Malignancy after gastrointestinal augmentation in childhood. Ther Adv Urol 2009;1:5-11. https://doi.org/10.1177/1756287209104163

34. Higuchi TT, Higuchi TT, Granberg CF, et al. Augmentation cystoplasty and risk of neoplasia: Fact, fiction, and controversy. J Urol 2010;184:2492-6. https://doi.org/10.1016/i.juro.2010.08.038

35. Cain MP, Rink RC, Yerkes EB, et al. Long-term followup and outcome of continent catheterizable vesicocstomy using the Rink modification. J Urol 2002;168:2583-5. https://doi.org/10.1016/S0022$5347(05) 64221-8$

36. Riachy E, Defoor WR, Reddy PP, et al. Endoscopic treatment with dextranomer/hyaluronic acid for persistent incontinence after continent urinary reconstruction. J Endourol 2015;29:137-40. https://doi.org/10.1089/end.2014.0326

37. Yerkes EB, Cain MP, King S, et al. The Malone antegrade continence enema procedure: Quality of life and family perspective. J Urol 2003;169:320-3. https://doi.org/10.1016/S0022-5347(05)64116-X

38. Chait PG, Shandling B, Richards HF. The cecostomy button. J Pediatr Surg 1997;32:849-51. https:// doi.org/10.1016/50022-3468(97)90634-5

39. Hoy NY, Metcalfe P, Kiddoo DA. Outcomes following fecal continence procedures in patients with neurogenic bowel dysfunction. J Urol 2013;189:2293-7. https://doi.org/10.1016/i.juro.2012.12.052

40. Heller MK, Gambino $S$, Church $P$, et al. Sexuality and relationships in young people with spina bifida and their partners. J Adolesc Health 2016;59:182-8. https://doi.org/10.1016/i.jadohealth.2016.03.037

41. Lee NG, Andrews E, Rosoklija I, et al. The effect of spinal cord level on sexual function in the spina bifida population. J Pediatr Urol 2015;11:142.el-6. https://doi.org/10.1016/i.jpurol.2015.02.010

42. Misseri R. Barriers to transition in young adults with neurogenic bladder. J Pediatr Urol 2016;12:259. https://doi.org/10.1016/i.purol.2016.04.050

43. Duplisea JJ, Romao RLP, MacLellan DL, et al. Urological followup in adult spina bifida patients: Is there an ideal interval? Urology 2016. [Epub ahead of print]. https://doi.org/10.1016/j.urology.2016.06.025

44. Husmann DA. Long-term complications following bladder augmentations in patients with spina bifida: Bladder calculi, perforation of the augmented bladder and upper tract deterioration. Transl Androl Urol 2016:5:3-11.

Correspondence: Dr. Peter D. Metcalfe, University of Alberta, Stollery Children's Hospital, Edmonton, AB, Canada; pmetcalf@ualberta.ca 